\title{
Uma Visão do Uso de MOOCs como Ferramenta de Capacitação para Docentes de Alunos com TEA
}

\section{A View of the Use of MOOCs as a Training tool for Teachers of Students with ASD}

\author{
Vanessa da Silva Balbino ${ }^{\mathrm{a}}$; Sérgio Crespo C. S. Pinto ; Ruth Maria Mariani Braz
}

\begin{abstract}
a'Universidade Federal Fluminense, Programa de Pós-Graduação Stricto Sensu em Diversidade e Inclusão. RJ, Brasil. bUniversidade Federal Fluminense, Programa de Pós-Graduação Stricto Sensu em Ciência, Tecnologia e Inclusão. RJ, Brasil. E-mail: sb.vanessa@gmail.com.
\end{abstract}

\begin{abstract}
Resumo
Este é um artigo que discute e analisa o uso dos Cursos Online Abertos Massivos - MOOC como ferramenta para docentes de alunos com Transtornos do Espectro do Autismo - TEA. No estudo buscou-se responder de que forma os cursos online, no formato MOOC podem ser utilizados como ferramentas em uma perspectiva inclusiva na capacitação de professores de alunos com TEA. Na presente pesquisa foi adotado o procedimento metodológico de revisão de literatura, com uma abordagem qualitativa e quantitativa. Utilizamos as bases de busca da Biblioteca Brasileira de Teses e Dissertações - BDTD, do Portal de Periódicos da Capes, do Google Acadêmico e da Scientific Eletronic Library Online- SciELO, com o objetivo de levantar os trabalhos publicados sobre a temática nos últimos cinco anos. Apontamos que os cursos MOOC são considerados ferramentas abertas, por serem gratuitos, bem como massivos, pois podem captar um número elevado de pessoas. Estes cursos podem ser divididos em módulos e na plataforma escolhida, são disponibilizados textos, vídeos, fóruns e exercícios. Concluímos que os MOOC, quando utilizados numa perspectiva inclusiva, por meio da formação continuada, podem servir de ferramenta de apoio aos professores que poderão explorar conteúdos e materiais valiosos para a aprendizagem de estudantes com TEA.
\end{abstract}

Palavras-chave: Cursos Online Massivos. Autismo. Formação Continuada.

\begin{abstract}
This is an article that discusses and analyzes the use of Massive Open Online Courses (MOOCs) as a tool for teachers of students with Autism Spectrum Disorders (ASD). In the study we tried to answer the following research question: How can online courses in MOOC format be used as tools from an inclusive perspective in the training of teachers of students with ASD? We used the search bases of the Brazilian Library of Theses and Dissertations (BDTD), of the Capes Periodical Portal, of Google Scholar and of the Scientific Eletronic Library Online (Scielo), with the objective of to survey the works published on the subject in the last 5 years. In this present research we adopted the methodological procedure of literature review with a qualitative and quantitative approach. As a result, we found that MOOCs courses are considered open tools, as they are free, as well as massive, as they can attract a large number of people. These courses can be divided into modules and on the chosen platform, texts, videos, forums and exercises are available. We conclude that MOOCs, when in an inclusive perspective, through continuing education, can serve as a support tool for teachers who will be able to explore valuable content and materials for the learning of students with ASD.
\end{abstract}

Keywords: Massive Online Courses. Autism. Continuing Education.

\section{Introdução}

Neste estudo, busca-se refletir sobre a importância da formação continuada docente, levando em consideração a relevância de arquiteturas pedagógicas e artefatos computacionais para o suporte a alunos com Transtorno do Espectro do Autismo - TEA.

O estudo tem como perspectivas contribuir para ampliar os horizontes relacionados à Educação Inclusiva, auxiliando os docentes e demais profissionais da Educação que atuam com autistas em suas estratégias voltadas para a aprendizagem desses indivíduos.

A pesquisa originou-se a partir das constantes indagações sobre a forma de inclusão de alunos autistas, tendo em vista que muitos dos problemas que justificam a "não inclusão" como argumento referem-se à falta de uma formação adequada para trabalhar com esses alunos em sala de aula.
Nesse sentido, criar meios alternativos para superar essas dificuldades é tarefa essencial dos professores, educadores e pesquisadores do assunto de forma geral. Isso irá resultar em professores mais preparados para lidar com alunos autistas, facilitando o processo de inclusão e o desenvolvimento da aprendizagem dos mesmos.

Inserindo-se nesse contexto, a formação continuada docente busca proporcionar diversos benefícios à medida que agrega conhecimentos, recursos e metodologias, visando caminhos alternativos ou inovadores para a inclusão de alunos com deficiência, objetivando atender às diferenças, combater o preconceito e entender à diversidade de forma ampla e abrangente no contexto educacional.

Entende-se que as tecnologias podem ser fortes aliadas dos professores no processo de ensino-aprendizagem desses alunos, porém o uso de recursos tecnológicos exige dos professores constantes atualizações, de forma que 
estes se apropriem de novos artefatos para aplicá-los no desenvolvimento dos seus conteúdos de ensino.

Sendo assim, é importante, no cenário brasileiro, identificar quais ferramentas computacionais estão disponíveis de forma gratuita na formação dos professores, para capacitá-los/ auxiliá-los visando à inclusão de alunos com TEA. Ambientes de EAD são uma boa opção, e, dentro dessa área, os MOOC são os que oferecem acesso gratuito e com flexibilidade de horário para que se possa realizar uma capacitação centrada na disponibilidade de tempo do professor.

Logo, artefatos computacionais, recursos tecnológicos, quando utilizados de forma adequada para capacitar professores de alunos com TEA, podem trazer diversos benefícios e estimular a aprendizagem desses alunos. Porém, o aprendizado de tais recursos e tecnologias depende de uma boa formação e possibilidades de capacitação do corpo docente.

Diante disso, a pesquisa tem como objetivo analisar e discutir como os Cursos Online Abertos Massivos - MOOC podem ser utilizados para capacitação do quadro docente no trato de alunos com TEA.

\section{Desenvolvimento}

\subsection{Metodologia}

A presente pesquisa está dividida em dois eixos basilares que são: o entendimento conceitual de MOOC e a aplicação dos MOOC na capacitação de professores que possuem alunos com TEA. Por meio da revisão sistemática da Literatura, buscou-se compreender esses dois eixos temáticos no contexto brasileiro.

O estudo teve como objetivo primário responder à seguinte questão de pesquisa: De que forma os cursos online, no formato MOOC, podem ser utilizados, como ferramentas, em uma perspectiva inclusiva, na capacitação de professores de alunos com TEA?

A partir da questão central, outras questões de pesquisa foram estabelecidas para facilitar a obtenção das respostas, conforme o quadro abaixo:

Quadro 1 - Questões de pesquisa e suas respectivas motivações

\begin{tabular}{|c|l|l|}
\hline ID & \multicolumn{1}{|c|}{ Questão de Pesquisa } & \multicolumn{1}{|c|}{ Motivação } \\
\hline QP1 & $\begin{array}{l}\text { Como os MOOC são } \\
\text { definidos e caracterizados? }\end{array}$ & $\begin{array}{l}\text { Entender as principais } \\
\text { características dos cursos } \\
\text { em formato massivo. }\end{array}$ \\
\hline QP2 & $\begin{array}{l}\text { Quais os benefícios e } \\
\text { desafios na condução de } \\
\text { um MOOC? }\end{array}$ & $\begin{array}{l}\text { Compreender quais os } \\
\text { benefícios e desvantagens/ } \\
\text { desafios na realização e } \\
\text { condução de um curso } \\
\text { nesse formato. }\end{array}$ \\
\hline QP3 & $\begin{array}{l}\text { Qual a relevância de de } \\
\text { recursos tecnológicos } \\
\text { para a prática pedagógica } \\
\text { docente? }\end{array}$ & $\begin{array}{l}\text { Mapear a importância e os } \\
\text { benefícios das tecnologias } \\
\text { quando aplicadas na prática } \\
\text { pedagógica docente. }\end{array}$ \\
\hline QP4 & $\begin{array}{l}\text { A utilização de tecnologias } \\
\text { traz impacto considerável } \\
\text { para o processo de ensino- } \\
\text { aprendizagem de alunos } \\
\text { com TEA? }\end{array}$ & $\begin{array}{l}\text { Compreender se o uso de } \\
\text { recursos tecnológicos traz } \\
\text { mudanças significativas no } \\
\text { aprendizado de alunos com } \\
\text { TEA. }\end{array}$ \\
\hline
\end{tabular}

Fonte: Dados da pesquisa.
A partir da definição das questões de pesquisa, foi possível definir os termos-chave que foram utilizados na busca. Estes foram: MOOC, Tecnologias, Autismo.

Para responder à questão central, bem como às questões secundárias do estudo, foram feitas buscas de publicações nas bases de dados da Biblioteca Brasileira de Teses e Dissertações - BDTD, do Portal de Periódicos da Capes, do Google Acadêmico e da Scientific Eletronic Library Online SciELO, a fim de levantar os trabalhos publicados sobre a temática nos últimos cinco anos. As leituras foram realizadas no período de setembro de 2019 a fevereiro de 2020. Nem sempre se obteve êxito na busca, tendo em vista que muitos dos artigos listados abordavam o assunto de forma muito abrangente ou muito superficial e sem relevância.

Os critérios de inclusão serviram para selecionar os artigos mais relevantes, assim como os critérios de exclusão serviram para descartar os artigos sem relevância para o estudo.

Desse modo, os critérios de inclusão foram: artigos mais relevantes; acesso livre ao documento; e respondem de forma clara e objetiva as questões da pesquisa.

Quanto aos critérios de exclusão, foram: artigos fora do escopo; trabalhos duplicados; que abordam tangencialmente a temática da pesquisa; trabalhos que não esclarecem as questões da pesquisa; e que não refletem aplicações do estudo no Brasil.

Durante as etapas da revisão sistemática da Literatura, os artigos foram categorizados conforme os critérios de inclusão: artigo que auxilia a conceituar e caracterizar os MOOC; que menciona os benefícios e desvantagens/desafios dos cursos MOOC; que descreve a importância dos recursos tecnológicos como meio de apoio às práticas pedagógicas dos docentes; e que relaciona como os diversos recursos digitais e aplicativos podem ser utilizados para a inclusão de alunos com TEA.

A primeira etapa para selecionar os trabalhos se deu por meio da leitura do seu título, resumo e palavras-chave. Todavia, mediante o número elevado de resultados retornados, esta primeira etapa foi dividida em duas subcategorias, em que a primeira consistiu na leitura do título e das palavras-chave e a segunda subcategoria na leitura do resumo. Já a segunda etapa consistiu na leitura da introdução e conclusão. A partir daí, foram selecionados aqueles que atendiam aos critérios de inclusão supracitados.

\subsection{Resultado}

A revisão sistemática da Literatura sintetizou conhecimentos que poderão ser utilizados para pensar práticas pedagógicas de inclusão educacional de alunos com deficiência, em especial, alunos com TEA.

As análises resultaram da revisão sistemática por meio de pesquisas bibliográficas. Foram identificados 1.244 trabalhos no total, levando em consideração as bases de dados pesquisadas. A partir desse levantamento, os trabalhos encontrados foram submetidos à primeira etapa do processo. 
A primeira etapa consistiu na leitura do título, do resumo e das palavras-chave. Os critérios de inclusão e de exclusão serviram como apoio para esta etapa. Após isso, 143 trabalhos foram considerados claros quanto às informações necessárias para os critérios de inclusão e de exclusão. Desse modo, 143 trabalhos foram selecionados e 1.101 trabalhos foram descartados para a revisão sem necessidade de submissão à etapa seguinte.

$\mathrm{Na}$ segunda etapa, 121 trabalhos apresentaram clareza suficiente e atenderam aos critérios de inclusão e exclusão. Desses, 8 trabalhos foram selecionados e 113 foram descartados sem a necessidade de submissão à segunda etapa da seleção. Todavia, os 22 trabalhos restantes, por não apresentarem clareza suficiente, passaram pela leitura da introdução e conclusão. Concluída a segunda etapa, 12 trabalhos foram selecionados e 10 foram descartados.

Sendo assim, 20 trabalhos estão embasando a presente pesquisa, sendo considerados relevantes quanto aos critérios de inclusão. Destes, dez trabalhos são provenientes do Google Acadêmico, oito provenientes da BDTD, um trabalho proveniente da Scielo e um proveniente do Portal de Periódico da Capes.

Os resultados encontrados e descritos abaixo fornecem respostas para as questões de pesquisa apontadas no presente estudo e sugerem propostas de novas investigações no que se refere a MOOC e à formação continuada docente na vertente inclusiva.

\subsection{Análise}

A primeira questão (QP1) tem como objetivo entender como os MOOC são definidos, bem como as principais características dos cursos em formato massivo e as categorias de classificação em que os MOOCs se dividem.

Os resultados apontam que $15 \%$ dos trabalhos selecionados trazem o conceito de MOOC, suas principais características e $5 \%$ apresentam as categorias de classificação em que estão divididos.

Em primeiro lugar torna-se necessário entendermos que os MOOC integram a Educação a Distância. A EAD consiste na modalidade de ensino-aprendizagem que ocorre em Ambientes Virtuais de Aprendizagem - AVA, por meio de tecnologias, permitindo que o professor e o aluno estejam em diferentes ambientes físicos.

Com o decorrer dos anos, a EAD trouxe muitos avanços para a Educação, potencializando o ensino, permitindo o desenvolvimento do pensamento crítico, tornando os alunos mais autônomos (tendo em vista que a presença simultânea do professor é dispensável) e, além disso há um constante equilíbrio entre a flexibilidade e a interação.

A partir da Lei de Diretrizes e Bases da Educação Nacional
(LDB 9394/96), regulamentada pelo Decreto 5.622/2005 ficou definida a qualidade da EAD no mesmo nível de outras modalidades de ensino.

Por volta do ano de 2000, iniciou-se a "geração da internet web". Foi a partir daí que houve a criação de cursos online, possibilitando o aprendizado em diferentes espaços físicos por várias pessoas simultaneamente.

O primeiro MOOC foi oferecido por George Siemens, Stephen Downes e Dave Cormier na Universidade de Manitoba, no Canadá, em 2008 (SOUZA; CYPRIANO, 2016). De acordo com Rodrigues et al. (2016), a partir do surgimento dos MOOC, os cursos nesse formato começaram a crescer e se expandir de forma considerável.

É nesse sentido que surgem os cursos MOOC em diferentes plataformas, tais como Coursera ${ }^{1}$ e Veduca ${ }^{2}$. Os MOOC surgem como forma de ampliar o modelo conectivista, possibilitando uma aprendizagem de forma colaborativa, como, por exemplo, o curso ofertado pelo Instituto Superior de Ciências da Informação e da Administração de Portugal ${ }^{3}$.

O termo MOOC passou a ser mais difundido a partir de 2007. De acordo com Pappano (2012), o grande crescimento dos MOOC fez com que 2012 fosse conhecido como o ano do MOOC.

Neste artigo, usamos a definição de McAuley et al. (2010, p.10) que mencionam:

Um MOOC é um curso online com a opção de registro gratuito e aberto, um currículo compartilhado publicamente, e os resultados em aberto. MOOCs integram redes sociais, recursos acessíveis online e são facilitados por lideranças na área de estudo. Mais significativamente, MOOCs são baseados no engajamento dos estudantes que auto-organizam sua participação de acordo com os objetivos de aprendizagem, conhecimento prévio, habilidades e interesses em comum.

Subbian (2013), assim como Andrade e Silveira (2016), consideram os MOOC como cursos de registro aberto, disponíveis nos AVA e que oferecem capacitação a um número extenso de participantes por meio de um currículo compartilhado. Siemens (2013), compreende os MOOCs como recursos para aprendizagem colaborativa por meio de tecnologias contemporâneas. Sendo que as tecnologias têm papel importante para favorecer a socialização por meio do ensino-aprendizagem.

Para Forno e Knoll (2013, p.183):

Diferentemente dos cursos tradicionais de Educação a Distância (EAD), os MOOCs são abertos, ou seja, podem ser acessados por qualquer pessoa conectada à internet, mediante sua inscrição em uma plataforma; não há critérios para a seleção de estudantes, exceto quando é indicada a necessidade de determinado conhecimento prévio e os cursos são majoritariamente gratuitos. Por essa ampla abrangência, os MOOCs são intitulados massivos, alcançando um grande número de pessoas. 
Ainda segundo Forno e Knoll (2013), os MOOC estão divididos em duas categorias distintas: O cMOOC e o xMOOC, tais nomenclaturas diferenciam os modelos pedagógicos dos cursos. O primeiro está baseado no conectivismo; os cursos nesse formato são desenvolvidos de forma mais informal e não dependem de uma instituição tradicional de ensino. Já no segundo, os formatos dos cursos são pré-definidos pelos professores da universidade que são figura central nessa categoria e não existe prioridade na interatividade entre os estudantes.

Entende-se que os cMOOCs estão relacionados à criatividade, autonomia, aprendizagem social em rede, já os xMOOCs relacionam-se a uma aprendizagem mais tradicional, sendo assim os cMOOCs são modelos conectivistas de aprendizagem, e os xMOOCs seguem o modelo behaviorista (DUBOSSON; EMAD, 2015). Ou seja, os cMOOCs têm foco na criação e geração do conhecimento, em que o professor atua como mediador e o aluno configura como participante ativo do processo de construção do conhecimento. Nesse modelo existe uma interação maior entre os alunos que também podem compartilhar materiais, potencializando o uso das tecnologias. Já os xMOOCs focam na duplicação do conhecimento, no qual o professor atua como figura central, detentor do conhecimento que é transmitido por ele e recebido pelos cursistas, porém a interatividade entre os alunos está em segundo plano. Em 2013, os MOOCs já se tornaram uma tendência mundial (FORNO; KNOLL, 2013).

Nesse mesmo contexto, Amado (2016, p.54) afirma que com o uso dos MOOC “[...] pretende-se reduzir barreiras no acesso à informação e ao diálogo, possibilitando o crescimento do conhecimento da sociedade".

Pessôa et al. (2016), referem-se aos MOOC como aprendizagem aberta, baseados no conectivismo, onde o conhecimento é construído e distribuído em redes. Segundo Maia e Carmo (2017), os MOOC são considerados massivos porque o professor pode atuar com um número de participantes maior do que o número de alunos de um curso presencial e como estratégias de educação para a cidadania e possuem algumas características que são: cursos participativos, desenvolvidos em rede, criativos e de qualidade.

Embora existam várias definições para os MOOC, Peglow, Geiger e Betamin (2015) explicam que em todos os conceitos existem três pontos em comum: são livres (abertos/ gratuitos), em larga escala (massivos) e simples (um professor que coordena as informações disponíveis no ambiente de aprendizagem.

Ainda nessa linha de raciocínio, estudos de Zaduski (2017) mostram que o ponto principal de um curso MOOC é a interação entre os participantes. Em seus estudos, os MOOC foram entendidos como um Ambiente Virtual de Aprendizagem em que o aluno cria, interage, compartilha, ou seja, constrói e transforma o conhecimento.

Silva e Marques (2015, p.234), complementam que o:
MOOCs são desenvolvidos em uma plataforma digital específica para serem abertos para todos que se interessarem, organizados com materiais e atividades a fim de promover a interação dos alunos através do estudo compartilhado e colaborativo, sem a obrigatoriedade do acompanhamento de professor/tutor (obrigatoriedade que há na modalidade de ensino a distância). As trocas entre os alunos são baseadas no estudo dos materiais disponibilizados por uma organização de ensino, mas o aluno define sua trajetória de aprendizagem.

Seguindo essa lógica de pensamento, cabe ressaltar o entendimento de Andrade e Silveira (2016) ao tratarem de recursos tecnológicos por meio de plataformas. Os pesquisadores frisam sobre a importância de o usuário ter um grau de conhecimento ainda que mínimo sobre ferramentas tecnológicas de acesso à internet.

Além de entender o conceito de MOOC, suas características e as categorias em que estão divididos, este estudo também propõe identificar quais as vantagens e desvantagens dos MOOC. Cabe ressaltar que os MOOC assumem papel de suma importância no tocante à formação continuada de professores, pois permitem aos mesmos reciclarem seus conhecimentos e adquirirem novas habilidades e competências.

Trazendo para o contexto da nossa pesquisa, podemos afirmar que os MOOCs podem ser desenvolvidos para capacitar docentes que atuam com alunos com TEA. Dado o exposto, de acordo com Almeida e Marques (2015), bem como com Medeiros e Bezerra (2016), a formação continuada configura-se como uma estratégia valiosa nas práticas pedagógicas dos docentes. Ou seja, além de compartilharem estratégias e experiências voltadas para a inclusão, a formação continuada por meio de um MOOC possibilitará aos docentes refletir sobre sua prática, servindo como uma rede colaborativa de aprendizagem.

A segunda questão de pesquisa (QP2) possui como propósito compreender quais os benefícios e os desafios/ desvantagens de se realizar um curso nesse formato. De acordo com os resultados obtidos, $20 \%$ dos trabalhos selecionados trazem as vantagens dos cursos em formato MOOC.

As leituras realizadas sobre o tema permitem-nos afirmar que a tecnologia por meio de recursos massivos online, como cursos de capacitação no formato MOOC, funciona como uma alternativa para suporte aos docentes de alunos com TEA. Os MOOCs eliminam barreiras que dificultam o aprendizado e porventura ainda existam em cursos tradicionais.

Ao realizar um curso de formação continuada via MOOC, os professores podem enriquecer as suas aulas com os conhecimentos adquiridos sobre vários temas e ao mesmo tempo se atualizarem profissionalmente.

De acordo com Papert (1994, p.106), “a implementação da informática na educação consiste basicamente de quatro ingredientes: o computador, o software selecionado, o professor capacitado a usar o computador no processo educativo, e o aluno". Ou seja, o professor assume o papel principal dentre os demais ingredientes, pois será o mediador do processo de ensino-aprendizagem e deverá usá-lo para criar 
espaços transdisciplinares, criando uma rede de significados entre as diferentes disciplinas do currículo escolar.

Sendo assim, torna-se necessário que os professores se apropriem das novas tecnologias online presentes na era digital, em especial por meio de cursos MOOC como forma de desenvolvimento pessoal e profissional, pois são recursos que possibilitam benefícios diversos para o aluno deficiente ou não.

Desse modo, levando em consideração os objetivos desta pesquisa, podemos pensar nos MOOC como sendo um importante instrumento de formação continuada para professores que atuam com alunos autistas.

Souza e Cypriano (2016) destacam os MOOC como forma de aperfeiçoamento e atualização sobre temas de interesse do usuário. Logo:

Os MOOCs representam experiências de aprendizagem realmente inovadoras. Vão além das experiências iniciais e limitadas de mudança na educação, como o OCW (Open Course Ware), baseadas ainda em objetos de aprendizagem isolados e sem pedagogias concretas associadas, e incluem não apenas mudanças na forma de compreender o conteúdo, mas também propostas metodológicas e novos papéis para os dinamizadores e participantes (HERNÁNDEZ, 2010, p.193).

Por ser online, o MOOC oferece certas vantagens, como, por exemplo, o acesso ao curso em qualquer lugar e em qualquer horário, a administração do tempo fica a critério do participante, possibilita a democratização da Educação, são flexíveis, permitem aprendizagens inovadoras, favorecem a aquisição de novas habilidades, compartilhamento de pensamentos e de materiais, são mais acessíveis do que os cursos tradicionais, oferecem conteúdos diversificados, favorecem o uso de novas tecnologias digitais na Educação, geralmente são totalmente gratuitos etc. Além disso, dependendo da instituição onde o curso for realizado, o aluno poderá obter um certificado de conclusão ao final do curso.

Dessa forma, a partir dos trabalhos estudados percebe-se que mediante o uso dos MOOC o aprendizado não fica mais restrito a quatro paredes de uma sala de aula e pode ocorrer por intermédio de outros suportes por meio das tecnologias digitais aplicadas na Educação, permitindo aprendizagens de forma mais dinâmica e flexível, impactando de forma positiva o papel dos professores.

Na visão de Wagner et al. (2016) os MOOC são cursos de curta duração com o intuito de oferecer uma formação inicial sobre determinado tema ou assunto, sendo considerados novas estratégias de formação por meio de recursos digitais.

Outro ponto relevante sobre os MOOC e que foi evidenciado nas leituras sobre o tema é que eles estão associados à teoria da aprendizagem do conectivismo, desenvolvida por George Siemens e Stephen Downes.
O conectivismo apresenta um modelo de aprendizagem que reconhece as mudanças tectônicas na sociedade, onde a aprendizagem não é mais uma atividade interna, individualista. $\mathrm{O}$ modo como a pessoa trabalha e funciona são alterados quando se utilizam novas ferramentas. O campo da educação tem sido lento em reconhecer, tanto o impacto das novas ferramentas de aprendizagem como as mudanças ambientais na qual tem significado aprender. $\mathrm{O}$ conectivismo fornece uma percepção das habilidades e tarefas de aprendizagem necessárias para os aprendizes florescerem na era digital. (SIEMENS, 2004, p.8).

Mattar(2013) também explica essa teoria da aprendizagem, na qual o aluno é capaz de se conectar, explorar, criar e avaliar os conteúdos é uma tentativa de ampliar o modelo conectivista para larga escala.

Existem diversos portais de tecnologia assistiva - TA disponíveis para os professores procurarem ferramentas que auxiliem este conectivismo e que proporcionem atendimento que auxilie os estudantes com TEA, como por exemplo destacamos: Association for the Advancement of Assistive Technology in Europe (AAATE) ${ }^{4}$, European Assistive Technology Information Network (Eastin) ${ }^{5}$, o Portal Nacional de Tecnologias Assistivas ${ }^{6}$ e o Banco Internacional de Objetos de Aprendizagens ${ }^{7}$.

De acordo com Weiss e Cruz (2001) o importante não é o conjunto de conhecimentos, mas o que esses conhecimentos possibilitam para novas aprendizagens. Sendo assim, os softwares quando utilizados numa perspectiva educacional subsidiam a prática pedagógica docente, tornam o conteúdo dinâmico, facilitando o aprendizado.

Littlejohn (2002) menciona que, oferecer um desenvolvimento profissional docente contínuo, aumenta as competências e, ao mesmo tempo, a eficácia de incluir novos métodos de ensino para o aprendizado dos alunos.

No que tange aos desafios/desvantagens dos cursos em formato massivo, $10 \%$ dos trabalhos estudados descrevem tais desvantagens. Léris et al. (2017), assim como Rodrigues et al. (2016) e Zancanaro, Nunes e Domingues (2016) citam como desvantagem a dificuldade de acompanhar e avaliar o aprendizado do aluno em cursos abertos e massivos, tendo em vista que podem ser captados um número elevado de participantes. Por outro lado, Zheng et al. (2016) apontam o risco de evasão como desvantagem. Esse risco deve-se ao fato de falta de motivação, falta de habilidade com as Tecnologias da Informação e Comunicação - TIC, além de carência por parte do aluno de uma interação constante com o professor. Já Fontana e Leffa (2018) destacam que neste formato de curso há pouca interatividade entre professor e aluno e falta de feedback entre os mesmos.

Tendo em vista os desafios mencionados, para que seja possível a análise da experiência de aprendizagem, é preciso

\footnotetext{
4 https://aaate.net/

5 http://www.eastin.eu/pt-pt/searches/Products/Index

$6 \mathrm{https}$ ://assistivaitsbrasil.wordpress.com/catalogo/

$7 \mathrm{http}: / /$ objetoseducacionais2.mec.gov.br/
} 
que seja feito um acompanhamento e interação com o aluno. Sendo assim, por tratar-se de um curso na modalidade EAD, é necessário que o aluno possua iniciativa, criticidade, saiba interagir e tenha autonomia.

Portanto, entende-se que a colaboração dos participantes do curso nos fóruns e nas tarefas propostas é a essência dos MOOC. Logo, pressupõe-se que os participantes tenham objetivos e interesses comuns de aprendizagem.

Quanto aos desafios já mencionados, Schmitt et al. (2015) expõem que, apesar das desvantagens existentes, os MOOC cresceram muito e, por tratar-se de um formato de curso flexível e inovador, suas vantagens se sobrepõe as desvantagens existentes.

Seguindo com a intenção de entender a relevância de recursos tecnológicos na Educação, a terceira questão de pesquisa (QP3) propõe mapear a importância e os benefícios das tecnologias quando aplicadas na prática pedagógica docente. A maioria dos trabalhos selecionados (25\%) traz resultados positivos nesse aspecto.

Silva e Marques (2015) explicam que por meio dos MOOCs podemos utilizar diferentes recursos audiovisuais como imagens, áudios, textos etc. Ou seja, trazendo essa ideia para o nosso estudo, entendemos que por intermédio dos cursos em formato massivo, os professores podem inovar suas aulas por meio de diversas metodologias e ao mesmo tempo reciclarem os seus conhecimentos, atualizando-se profissionalmente.

Dado o exposto, Gonçalves e Gonçalves (2015), assim como Souza e Cypriano (2016) entendem que os MOOC favorecem a formação continuada de professores tendo como foco o aprendizado colaborativo e o desenvolvimento de várias competências, o que acarreta impacto positivo nas áreas de Educação e tecnologia.

Desse modo, utilizando estratégias diversificadas por meio de cursos no formato MOOC, os professores têm a oportunidade de dinamizar a aprendizagem dos alunos, principalmente daqueles que possuem alguma necessidade educacional especial, incluindo os alunos com TEA.

Entendemos que a sala de aula deverá ser um ambiente de aprendizagem cooperativo, onde o professor atua como mediador, orientador e o aluno torna-se o centro de todo o processo sendo responsável pela produção do seu próprio conhecimento.

Conforme Citelli (2000, p.7), “a escola não deve temer nem subestimar o seu diálogo com os meios de comunicação e o uso das tecnologias [...] Não vejo os meios de comunicação como instrutores, quero pensá-los como produtores do conhecimento".

Sendo assim, podemos inferir que as tecnologias presentes na modernidade, como, por exemplo, os softwares educacionais, não substituem os professores, mas servem para subsidiar a prática pedagógica, tornar o aprendizado mais dinâmico e facilitar o aprendizado de todos os alunos, principalmente dos alunos com necessidades educacionais especiais.

No contexto educativo, os MOOCs representam um importante auxílio nos processos de ensino e aprendizagem proporcionando condições para a criação de redes e comunidades virtuais que favorecem a interação e colaboração entre os intervenientes, através da troca, partilha e redistribuição de conhecimentos (BAETA, 2016, p.1).

Diante dos dados mencionados, no que diz respeito às novas tecnologias aplicadas na Educação, considera-se relevante pensar nas possibilidades de sua aplicação e inserção nas práticas pedagógicas docentes.

Todavia, apesar dos avanços que as TIC trouxeram para a Educação, ainda existem inúmeros desafios relacionados à formação de professores e uma alternativa para solucionar o problema em um curto prazo de tempo é a Educação a Distância.

De acordo com Libâneo (1991, p.173):

Os professores precisam dominar com segurança esses meios auxiliares de ensino, conhecendo e aprendendo a utilizá-los. O momento didático adequado de utilizá-los vai depender do trabalho docente prático, no qual se adquirirá o efeito traquejo na manipulação do material didático.

Logo, torna-se necessário refletirmos sobre as necessidades formativas docentes, em especial nas necessidades relacionadas à inclusão de alunos com TEA. Não basta apenas inserir o computador dentro das escolas, deve-se pensar nos benefícios que os professores terão ao inserir recursos tecnológicos computacionais em suas aulas e, nesse sentido, torna-se necessário planejar e investir na formação continuada docente, pois sem capacitação e sem preparo adequado para uso desses recursos não haverá sucesso nesse processo e o professor continuará sem saber como utilizar as ferramentas tecnológicas a seu favor.

Dado o contexto, já visto na QP3, é notório que as diversas tecnologias presentes no mundo são de suma importância, porém, sem dúvida alguma, a internet, também denominada rede mundial de computadores, torna-se um diferencial se sobrepondo as demais.

A tendência global é que a internet continue se expandindo e, com isso, sejam ampliados os benefícios que ela proporciona em todas as áreas do saber. Desse modo, observase a necessidade de os profissionais da Educação buscarem o seu aperfeiçoamento por meio da formação continuada levando em consideração as novas tecnologias aplicadas na Educação. Entende-se, pois, que os cursos oferecidos na modalidade a distância são meios para facilitar a capacitação docente e atualmente tornaram-se uma tendência em várias universidades no Brasil e no mundo.

Podemos inferir que os MOOC apresentam um novo cenário para a EAD no que tange à interatividade entre os participantes que possuem o aprendizado como objetivo central. Os recursos massivos online por meio de um MOOC representam mudanças no processo de aprendizagem online, permitindo que os professores que atuam com alunos com 
TEA construam de forma coletiva os seus conhecimentos, colaborando para a disseminação de tais conhecimentos para demais profissionais que atuam com estudantes com autismo.

A última questão de pesquisa (QP4) propõe compreender os impactos dos recursos tecnológicos no processo de aprendizagem de alunos com TEA. Ou seja, considerando que alunos com deficiência possuem dificuldades no aprendizado, a presente questão de pesquisa busca analisar se as tecnologias aplicadas na Educação podem impactar positivamente e facilitar o processo de ensino-aprendizagem de alunos com TEA. Nesta questão, foram selecionados 25\% dos trabalhos analisados.

Diversos estudos apontam que as tecnologias digitais podem contribuir para o desenvolvimento de crianças com TEA e nesse contexto também se enquadram os jogos educativos eletrônicos e diversos outros dispositivos. Os estudos de Monte (2015); Coutinho, Bez e Passerino (2014); Passerino, Avila e Bez (2010) e Franciscatto et al. (2016), por exemplo, analisaram o Sistema de Comunicação Alternativa para Letramento de pessoas com Autismo, o SCALAWEB, que foi desenvolvido em 2009.

Atualmente, existem versões disponíveis na web para celulares android e tablets. Com o uso do sistema SCALAWEB, os sujeitos aprendem participando de forma interativa, autônoma e criativa.

Outra ferramenta tecnológica importante para a aprendizagem de alunos com TEA é o EDUKITO. Esse ambiente virtual foi pesquisado por Passerino (2005), de modo que tal ambiente trabalha por projetos e o aluno pode se inscrever em vários projetos. O objetivo do EDUKITO é analisar o grau de interação social e de mediação de indivíduos com TEA.

Entendemos, pois, que as tecnologias digitais proporcionam transformações relevantes nas áreas afetivas, cognitivas, emocional e na comunicação de crianças com TEA.

Desse modo, jogos virtuais, assim como outros aplicativos e recursos tecnológicos diversos podem ser utilizados para ensinar e aprender de forma inclusiva. Afinal, estimulam habilidades como coordenação motora, raciocínio lógico, concentração, percepção espacial, dentre outros nos alunos com TEA.

Alunos com deficiência usando tecnologias assistenciais podem se beneficiar enormemente dos MOOCs, não apenas porque permitem atividades de aprendizagem a distância e flexíveis, mas também porque ajudam os alunos com deficiências a acessar recursos que de outra forma apresentariam barreiras significativas a eles. (RODRIGO, 2014, p.114).

Ao realizar um MOOC com intuito de formação continuada que apresente aplicativos e softwares que podem ser utilizados no aprendizado de estudantes com TEA, tais como as ferramentas mencionadas no estudo em tela, estaremos capacitando os professores para auxiliarem os seus respectivos alunos. Além disso, a interação e a troca de experiência entre os docentes participantes possibilitarão a aquisição de conhecimentos valiosos. Assim, entendemos que os cursos MOOC são considerados como inclusivos; portanto, devem ser utilizados no contexto educacional, em especial no ensino de alunos com TEA.

Podemos afirmar que, com o advento da internet, a difusão do conhecimento tornou-se mais abrangente. Desse modo, recursos tecnológicos, quando utilizados de forma adequada para alunos com TEA (como por exemplo o computador atuando como uma tecnologia assistiva voltada para a comunicação alternativa), podem trazer diversos benefícios e estimular a aprendizagem desses alunos.

A evolução da internet e das tecnologias da informação e comunicação (TIC) têm possibilitado a participação ativa do usuário como leitor e produtor de conteúdo, e favorecido o aprendizado contínuo. Este aprendizado não ocorre somente nas escolas e universidades, mas também em outros contextos, como nas redes sociais, através de acesso a tutoriais, pesquisas e MOOCs, (Massive Open Online Courses - Cursos Abertos Online Massivos). (SILVA, 2014, p.121).

Segundo Moran (2015), as ferramentas disponíveis nos Ambientes Virtuais de Aprendizagem (AVA) favorecem o aprendizado e as práticas educacionais. $\mathrm{O}$ autor entende que as tecnologias ampliam os espaços de aprendizagem favorecendo práticas inovadoras.

Levando em consideração os aspectos relacionados à globalização:

É importante perceber que as tecnologias fazem parte do cotidiano das pessoas. Pois costumamos utilizá-las em diferentes contextos e momentos do nosso dia a dia, por inúmeros motivos, bem como para nos comunicar, nos mantermos informados, realizarmos trabalhos e pesquisas, entre outros. Dessa forma, compreende-se que as tecnologias digitais têm sido consideradas uma necessidade no mundo em que vivemos e, por este motivo, o uso destes instrumentos tem sido cada vez mais comum. (SANTOS; SCHWANKE; MACHADO, 2017, p.131)

Nesse contexto, Alves, Pereira e Viana (2017) entendem que o ciberespaço possui um caráter democrático e inclusivo e explicam que o espaço virtual permite-nos romper paradigmas, barreiras e fronteiras entre saberes e seres.

Cabe ressaltar que devido ao comprometimento da comunicação e da interação social, grande parte das crianças com autismo tendem a aprender com mais facilidade por meio de recursos visuais e audiovisuais. Os recursos audiovisuais facilitam o aprendizado dos alunos com TEA, pois acabam aproximando a comunicação do sentido real.

Sendo assim, materiais tecnológicos visuais e os diversos recursos tecnológicos digitais presentes na era atual estimulam a área cognitiva, sensorial e sócio emocional dos autistas, sendo muito importante que tais recursos sejam utilizados pelos professores para possibilitar uma efetiva inclusão dos educandos com TEA.

Estudos de Almeida et al. (2019) também apresentam uma outra ferramenta digital tão relevante para o desenvolvimento 
da aprendizagem de indivíduos com TEA quanto as ferramentas já mencionadas. Tal ferramenta consiste no game MOTIVA $E d u c$ e tem por objetivo auxiliar no desenvolvimento de competências cognitivas e outras habilidades em indivíduos com autismo.

Pesquisadores, tais como, Yakubova, Hughes e Shinaberry (2016); Carvalho e Nunes (2016); Santos, Breda e Almeida (2017) frisam que as tecnologias digitais são estratégias relevantes no processo de aprendizagem de alunos com TEA, tendo em vista que tais tecnologias facilitam a comunicação e consequentemente a interação desses estudantes.

No tocante ao aprendizado por meio de recursos tecnológicos, Caram e Bizelli (2014, p.201), afirmam:

Algo muito questionado em relação a EAD é o fato de não possuir necessariamente a presença física de um professor em sala de aula. Porém isso não diminui em nada a eficácia da EAD no que diz respeito ao processo de ensino-aprendizagem. Pelo contrário, a capacidade do professor de entender, mediar e estimular os alunos através das novas tecnologias deve ser ainda maior. Os esforços de ambas as partes torna-se essencial para que a transmissão de conhecimento aconteça de forma eficaz.

Diante dos dados mencionados, pode-se afirmar que os estudos analisados apontam o uso das tecnologias digitais como ferramentas valiosas para o desenvolvimento de atividades educacionais na vertente inclusiva.

Alunos com necessidades especiais necessitam de recursos que os coloquem o mais próximo possível de sua realidade. Sendo assim, dado o contexto da presente pesquisa, os cursos em formato MOOC, além de possibilitar a capacitação docente, podem impactar positivamente o aprendizado de alunos com autismo.

Entendemos, pois, que as ferramentas tecnológicas digitais possibilitam o desenvolvimento da autonomia, da coordenação motora, auxiliam no aprendizado da leitura e da escrita de alunos com TEA, dentre outros benefícios.

Logo, podemos afirmar que as diversas tecnologias digitais, tais como jogos e aplicativos, quando utilizados no cenário educacional, visando à inclusão, produzem um impacto significativo no processo de aprendizagem de alunos com necessidades educacionais especiais, possibilitando o desenvolvimento de competências e habilidades, inclusive em alunos com TEA.

No que tange aos MOOC em si, notamos que há necessidade de pesquisas que analisem e discutam o papel de democratização trazido pelos MOOC, tendo em vista tratar-se de um tema que ainda é pouco explorado no Brasil. De igual modo, devido à escassez de pesquisas voltadas para a capacitação de professores, visando à mediação por intermédio de tecnologias, torna-se necessário que estudos sobre a formação continuada docente por meio de cursos MOOCs sejam desenvolvidos, objetivando tornar esse recurso uma apropriação dos professores, como forma de ampliar as suas respectivas estratégias pedagógicas para a inclusão de alunos com necessidades educacionais especiais.

\section{Conclusão}

É possível verificar a fundamental importância que os docentes e demais profissionais que atuam com indivíduos autistas busquem se aperfeiçoar por meio da formação continuada na vertente inclusiva, para que sejam elaboradas estratégias e criadas possibilidades de uma efetiva inclusão por intermédio do uso de recursos tecnológicos.

Nesse contexto, a tecnologia presente na modernidade é parte acessória para contribuir e auxiliar os docentes em suas práticas pedagógicas, rompendo com métodos únicos e tradicionais de ensino. É necessário unir as diversas técnicas e ferramentas pedagógicas existentes para tornar os ambientes virtuais mais interativos e interessantes, de modo que os cursistas interajam entre si e se sintam motivados e engajados.

É dentro dessa lógica que vários cursos no formato MOOC chegam a universidades brasileiras para facilitar a disseminação de conteúdos diversos para a sociedade, incluindo docentes e demais educadores nesse processo.

$\mathrm{O}$ estudo tem como perspectivas contribuir para ampliar os horizontes relacionados à Educação Inclusiva, auxiliando os docentes e demais profissionais da Educação que atuam com autistas em suas estratégias voltadas para a aprendizagem desses indivíduos, proporcionando metodologias pedagógicas ativas e diversificadas, favorecendo uma educação online interativa e participativa.

Dado o contexto, podemos afirmar que os recursos tecnológicos existentes são ferramentas que visam a complementar e aumentar a qualidade do ensino, melhorando a comunicação de alunos com TEA e facilitando o trabalho docente.

Por meio dos recursos tecnológicos aplicados na Educação, o professor enriquece o processo de ensino junto com outros recursos disponíveis. Por isso, é de suma importância que os docentes busquem constante atualização e capacitação por intermédio dos recursos tecnológicos existentes na atualidade, pois, além de se capacitar, criarão possibilidades de equidade entre os educandos como forma de uma efetiva inclusão.

Entendemos, assim, que os artefatos computacionais presentes na modernidade auxiliam, mas não substituem o professor, sendo este o mediador de todo o processo educativo, a partir do qual estes recursos permitem a construção do conhecimento de forma criativa, crítica e autônoma.

\section{Referências}

ALMEIDA, D.S.; MARQUES, P. F. MOOCs: uma análise das experiências pioneiras no Brasil e Portugal - constatações e limitações. In: CONGRESSO INTERNACIONAL ABED DE EDUCAÇÃO A DISTÂNCIA. Bento Gonçalves, 2015. Anais... 2015. Disponível em: http://www.abed.org.br/congresso2015/ anais/pdf/BD_215.pdf. Acesso em: 13 out. 2019.

ALMEIDA, G.K.F.C. et al. MOTIVAEduc: um game baseado na metodologia ABA para a auxiliar na aprendizagem de crianças autistas. Olhares Trilhas, v.21, n.1, p.111-122, 2019. doi: https:// doi.org/10.14393/OT2019v21.n.1.46264.

ALVES, M.D.F.; PEREIRA, G.V.; VIANA, M.A.P. Tecnologia 
assistiva na perspectiva da educação inclusiva: o ciberespaço como lócus de autonomia e autoria. Laplage Rev., v.3, n.2, p.159-169, 2017. doi: https://doi.org/10.24115/S24466220201732347p.159-169.

AMADO, C.B.O.P. Segurança na internet para encarregados de educação: desenvolvimento de um MOOC. Lisboa: Universidade de Lisboa, 2016.

ANDRADE, M.V.M.; SILVEIRA, I.F. Panorama da aplicação de Massive Open Online Course (MOOC) no ensino superior: desafios e possibilidades. EAD em Foco, v.6, n.3, p.101-114, 2016. doi: https://doi.org/10.18264/eadf.v6i3.392.

BAETA, P.I.P. MOOCs desenvolvidos no ensino superior português: análise de modelos pedagógicos e estratégias de funcionamento utilizados. Lisboa: Universidade de Lisboa, 2016.

BRASIL. Ministério da Educação. Lei de Diretrizes e Bases da Educação Nacional, LDB nº 9394, de 20 de dezembro de 1996. Estabelece as diretrizes e bases da educação nacional. Brasília: MEC, 1996.

CARAM, N.R.; BIZELLI, J.L. Aspectos da regulação sobre o ensino a distância no Brasil. Rev. Online Pol. Gestão Educ., n.17, p.200-209, 2014. doi: https://doi.org/10.22633/rpge.v0i17.9367.

CARVALHO, O.M.F.; NUNES, L. R. Possibilidades do uso de jogos digitais com criança autista: estudo de caso. In: CAMINHA, V. L. et al. Autismo: vivências e caminhos. São Paulo: BLUCHER, 2016. p.77-90.

CITELli, A.O. Comunicação e educação: a linguagem em movimento. São Paulo: SENAC, 2000.

COUTINHO, K.S.; BEZ, M.R.; PASSERINO, L.M. Análise de contexto em interações com o SCALA Tablet mediando a comunicação de alunos incluídos com Autismo. Rev. Inform. Educ. Teor. Prática, v.17, n.1, p.221-231, 2014. doi: https://doi. org/10.22456/1982-1654.43423.

DUBOSSON, M.; EMAD, S. The Forum Community, the Connectivist Element of an xMOOC. Univ. J. Educ. Res., v.3, n.10, p.680-690, 2015. doi: https://doi.org/10.13189/ujer.2015.031004.

FONTANA, M.V.L.; LEFFA, V.J. MOOCs para o Ensino de Línguas: um estudo em call desde uma perspectiva conectivista. Alfa Rev. Linguística, v.62, n.1, p.75-89, 2018. doi: http://doi. org/10.1590/1981-5794-1804-4.

FORNO, J.P.D.; KNOLL, G.F. Os MOCCs no mundo: um levantamento de cursos online abertos massivos. Rev. Nuances, v.24, n.3, p.178-194, 2013. doi: http://dx.doi.org/10.14572/ nuances.v24i3.2705.

FRANCISCATTO, R. et al. Sistema SCALAWEB: busca avançada. Teknos, v.16, n.1, p.37-48, 2016. doi: https://doi. org/10.25044/25392190.801.

GONÇALVES, V.; GONÇALVES, B.M.F. Avaliação de plataformas para criação e distribuição de MOOC para a formação contínua de professores. In INTERNATIONAL CONFERENCE ON INNOVATION DOCUMENTATION AND TEACHING TECHNOLOGIES. Valencia: Universidad Politecnica de Valencia, 2015.

HERNÁNDEZ, D.R. Un mundo de medios sin fin: cambios en aprendizaje, Facebook y la apoteosis de las aplicaciones expresivas. In: PISCITELLI, A.; ADAIME, I.; BINDER, I. El Proyecto Facebook y la posuniversidad: sistemas operativos sociales y entornos abiertos de aprendizaje. Dialnet: Universidad de la Rioja, 2010. p.183-202.

LÉRIS, D. et al. Validation of indicators for implementing and adaptive platform for MOOCs. J. Comp. Hum. Behavior, v.72, p.783-795, 2017. doi: https://doi.org/10.1016/j.chb.2016.07.054.
LIBÂNEO, J. C. Didática: coleção magistério. São Paulo: Cortez, 1991.

LITTLEJOHN, A. H. Improving Continuing professional development in the use of ICT. J. Comp. Assisted Learning, v.18, n.2, p.166-174, 2002. doi: https://doi.org/10.1046/j.02664909.2001.00224.x.

MAIA \& CARMO, T. Como se aprende num MOOC? Rev. UIIPS, v.5, n. 2, p.198-210, 2017.

MATTAR, J. Aprendizagem em ambientes virtuais: teorias, conectivismo e MOOCs. Teccos, n.7, p.20-40, 2013.

MCAULEY, A. et al. The MOOC Model for Digital Practice. University of Prince Edward: Island through the Social Sciences and Humanities Research Council's, 2010. Disponível em: https://www.oerknowledgecloud.org/archive/MOOC_Final.pdf. Acesso em: 24 set. 2019.

MEDEIROS, L.M.B.; BEZERRA, C.C. Algumas considerações sobre a formação continuada de professores a partir das necessidades formativas em novas tecnologias na educação. In: SOUZA, RP. et al. Teorias e práticas em tecnologias educacionais. Campina Grande: EDU EPB, 2016. p.17-37.

MONTE, B.T. Por trás do espelho de Alice: narrativas visuais como estratégias de inclusão de crianças com Transtorno do Espectro do Autismo. Porto Alegres: Universidade Federal do Rio Grande do Sul, 2015

MORAN, J.M. Mudando a educação com metodologias ativas. In: SOUZA, C.A.; MORALES, O.E.T. Convergências midiáticas, educação e cidadania: aproximações jovens. Ponta Grossa: UEPG/PROEX, 2015. p.15-33.

PAPERT, S. A máquina das crianças: repensando a escola na era da informática. Porto Alegre: Artmed, 1994.

PAPPANO, L. The Year of the MOOC. New York Times, 2012. Disponível em: https:/www.nytimes.com/2012/11/04/education/ edlife/massive-open-online-courses-are-multiplying-at-a-rapidpace.html. Acesso em: 11 set. 2019.

PASSERINO, L.M. Pessoas com autismo em ambientes digitais de aprendizagem: estudo dos processos de interação social e mediação. Tese. (Doutorado em Informática na Educação) Universidade Federal do Rio Grande do Sul, Porto Alegre, 2005.

PASSERINO, L.M.; AVILA, B.G.; BEZ, M.R. SCALA: um sistema de comunicação alternativa para o letramento de pessoas com autismo. Rev. Novas Tecnol. Educ., v.8, n.2, 2010. doi: https://doi.org/10.22456/1679-1916.15224.

PEGLOW, T.S.; GEIGER, V.; BETAMIN, V.S. Os MOOCs: um novo recurso de aprendizagem na educação a distância. Rev. Cesuca Virtual, v.2, n.4, p.58-72, 2015.

PESSÔA, T. et al. O MOOC Escrita Criativa: "a outra tradição": uma experiência pedagógica inovadora na Universidade de Coimbra. Amb. Virtuais Ensino Superior, v.3, p.69-90, 2016.

RODRIGO, C. Acessibility in language MOOCs. In: MARTÍNMONJE, E.; MADERA, B. Language MOOCs: providing learning. transcending boundaries. Berlin: De Gruyter, 2014. p.106-126.

RODRIGUES, R. L. et al. Discovery engagement patterns MOOCs through cluster analysis. IEEE Latinam. Transac., v.14, n.9, p.4129-4135, 2016. doi: https://doi.org/10.1109/ TLA.2016.7785943.

SANTOS, M.I.; BREDA, A.; ALMEIDA, A.M. Design approach of mathematics learning activities in a digital environment for children with autism spectrum disorders. Educ. Technol. Res. Develop., v. 65, p.1305-1323, 2017. doi: https://doi.org/10.1007/ s11423-017-9525-2. 
SANTOS, P.K.; SCHWANKE, C.; MACHADO, K.G.W. Tecnologias digitais na educação: possibilidades para o desenvolvimento da educação para a cidadania global. Educ. Escrito, v.8, n.1, p.129-145, 2017. doi: https://doi. org/10.15448/2179-8435.2017.1.27674.

SCHMITT, S.P. et al. Os MOOCS na EAD. Rev. Cesuca Virtual, v.2, n.3, p.214-221, 2015.

SIEMENS, G. Conectivismo: uma teoria de aprendizagem para a idade digital. 2004. Disponível em: http://usuarios.upf. br/ teixeira/livros/conectivismo[siemens].pdf. Acesso em: 25 jan. 2020.

SIEMENS, G. Massive open online courses: innovation in education? 2013. Disponível em: https:/www. oerknowledgecloud.org/archive/pub_PS_OER-IRP_web. pdf\#page $=31$. Acesso em: 25 jan. 2020 .

SILVA, P.G.; MARQUES, P.F. MOOC como possibilidade de Ensino e Aprendizagem em cultura digital. In: TISE CONGRESSO INTERNACIONAL DE INFORMÁTICA EDUCATIVA, Santiago, Chile. 2015. Disponível em: http:// www.tise.cl/volumen11/TISE2015/TISE\%202015.pdf. Acesso em: 13 jan. 2020.

SILVA, S. MOOC como ambiente de aprendizagem. Rev. Sinergia, v.15, n.2, p.121-125, 2014.

SIMÕES, A. Avaliação de sites de matemática e implicações na prática docente: um estudo no $3^{\circ} \mathrm{CEB}$ e no Secundário. Dissertação. (Mestrado em Educação) - Universidade do Minho: Braga, 2005.

SOUZA, R.; CYPRIANO, E.F. MOOC: uma alternativa contemporânea para o ensino de astronomia. Ciênc. Educ., v.22, n.1, p.65-80, 2016. doi: https://doi.org/10.1590/1516731320160010005 .
SUBBIAN, V. Role of MOOCs in integrated STEM education: a learning perspective. In: IEEE INTEGRATED STEM EDUCATION CONFERENCE (ISEC), Princeton. 2013. doi: https://doi.org/10.1109/ISECon.2013.6525230

WAGNER, R. et al. SolAssist Learning: formação em tecnologias assistivas através de um MOOC e uma biblioteca virtual de soluções assistivas. Rev. Bras. Inform. Educ., v.24, n.3, p.62-74, 2016. doi: http://dx.doi.org/10.5753/rbie.2016.24.3.62.

WEISS, A.M.L; CRUZ, M.L.R.M. A informática e os problemas escolares de aprendizagem. Rio de Janeiro: DP\&A, 2001.

YAKUBOVA, G.; HUGHES, E.M.; SHINABERRY, M. Learning with technology: video modeling with concrete-representationalabstract sequencing for students with autism spectrum disorder. $J$. Autism Develop. Dis., v.46, p.2349-2362, 2016. doi: https://doi. org/10.1007/s10803-016-2768-7.

ZADUSKI, J.C.D. Aprendizagem e Interação em um ambiente de educação não formal: a Unesp Aberta e as potencialidades dos MOOCs. Dissertação (Mestrado em Educação) - Universidade Estadual Paulista, Presidente Prudente, São Paulo, 2017.

ZANCANARO, A.; NUNES, C.S.; DOMINGUES, M.J.S Free platforms for providing MOOCs: mapping and assessment requirements. In: CONTECSI - INTERNATIONAL CONFERENCE ON INFORMATION SYSTEMS \& TECHNOLOGY MANAGEMENT, p.3660-3673, 2016. doi: https://doi.org/10.5748/9788599693124-13CONTECSI/PS4154.

ZHENG, S. et al. Ask the instructors: motivations and challenges of teaching massive open online courses. CSCW 16: PROCEEDINGS OF THE 19TH ACM CONFERENCE ON COMPUTER-SUPPORTED COOPERATIVE WORK \& SOCIAL COMPUTING, 2016. doi: https://doi. org/10.1145/2818048.2820082. 